\title{
LOW NOISE LOW POWER READOUT CIRCUIT FOR SOFT X RAY DETECTION
}

\author{
A. Cerdeira-Estrada ${ }^{1}$, A. De Luca², A. Cuttin³ \& R. Mutihac4. \\ ${ }^{1}$ Telital Spa, Trieste Italy, alberto.cerdeira@telit.net \\ ${ }^{2}$ Depto. de Ingeniería Eléctrica, Sección de Computación, CINVESTAV-IPN, México D. F., \\ ${ }^{3}$ Laboratory of Microprocessors, ICTP-INFN, Trieste Italy. \\ ${ }^{4}$ University of Bucharest, Electronic Department, Romania. \\ Received September $18^{\text {th }} 2000$ and accepted August $26^{\text {th }} 2002$
}

\section{ABSTRACT}

A new low power CMOS ASIC for the detection of X-rays was optimized for low power and low noise. Theoretical calculations and optimizations are presented and compared with experimental results. Noise as low as $120+25 \cdot C_{\text {in }}$ [pF] ENC rms was obtained including a silicon detector of $1.3 \mathrm{pF}$ and $0.3 \mathrm{nA}$ of leakage. The power consumption is less than $100 \mathrm{~W}$. Other circuit parameters are also shown.

KEYWORDS: Charge sensitive amplifiers, Equivalent noise charge, Sharper.

\section{INTRODUCTION}

During the last years, an important effort has been dedicated to the development of electronics circuits for nuclear radiation measurements using crystalline and amorphous silicon diodes detectors. Recently we reported [1] a full custom integrated circuit designed for X-ray photon detection in a new system approach to digital radiography. For pixel array architectures, a big amount of pixels is needed to obtain high resolution and thus low power consumption and small area per channel are required in the readout circuits. Detection of soft $X$-ray used in medical applications also requires high gain preamplifiers and very low overall noise in the circuit. In this paper we present calculations and experimental results obtained for low noise optimization, while maintaining low power consumption and other required parameters.

\section{READOUT CIRCUIT CHARACTERISTICS}

The front end of the readout circuit consists of a Charge Sensitive Amplifier (CSA), designed to integrate the charge collected at the detector during a period of time much bigger than its collection time in order to create a voltage pulse at the output of the circuit. CSA are low bad pass filters with an integration time mainly dependent on the output impedance, peak voltage and feedback capacitor. Their noise is mainly due to the high transconductance input MOS transistor. To cut this unnecessary noise a narrow band filter called Shaper must be included and its parameters optimized to achieve required signal to noise ratio. JFTET transistors have less noise than MOS, but are difficult to implement using standard CMOS technology employed in our circuit.

Fig. 1 shows the first two blocks of the ASIC corresponding to the CSA and SHP, designed to obtain a maximum output voltage swing of $3 \mathrm{~V}$, a shaping time less than $5 \mu \mathrm{s}$ for a capacitive load of $20 \mathrm{pF}$, a power consumption less than $100 \mu \mathrm{W}$ and a single voltage supply of $5 \mathrm{~V}$, so it can be used in portable systems, space and big matrix detectors. The calculated gain was $3446 \mathrm{mV} / \mathrm{fC}$, to allow detection of charges above 400 electrons, if noise is 
maintained below 200 electrons. The detector used in [1] had a capacitance of $C_{d}=1.3 p F$ and diode leakage of $I s=0.35 \mathrm{nA}$. Fig. 2 shows the schematics of the detector diode with an AC coupling to the readout circuit.

\section{NOISE OPTIMIZATION}

Noise was theoretically minimized optimizing the detector bias resistor $R_{\text {sas, }}$ the input transistor transconductance $g_{m i}$, and the shaping time of the filter $\tau$. The noise in the circuit, expressed by the Equivalent Noise Charge (ENC) at the input, was calculated through equations [2,3]:

$$
\begin{gathered}
E N C_{d}=\sqrt{\left(2 q I_{L}+\frac{4 k T}{R_{\text {bias }}}\right) * \tau * \frac{(1.57 * 7.39)}{q^{2} 4 \pi}} \\
\text { ENC }_{\text {th }}=\sqrt{\frac{8}{3} k T \frac{1}{g_{m}} \frac{C_{t}^{2}}{q^{2} 4 \pi \tau}(1.57 * 7.39)} \\
E^{2} C_{f}=\sqrt{\frac{K_{f}}{C_{o x}{ }^{2} W L} \frac{C_{t}^{2}}{q^{2} 2}(1.57)}
\end{gathered}
$$

where detailed description of the parameters and their values are shown in Table I.

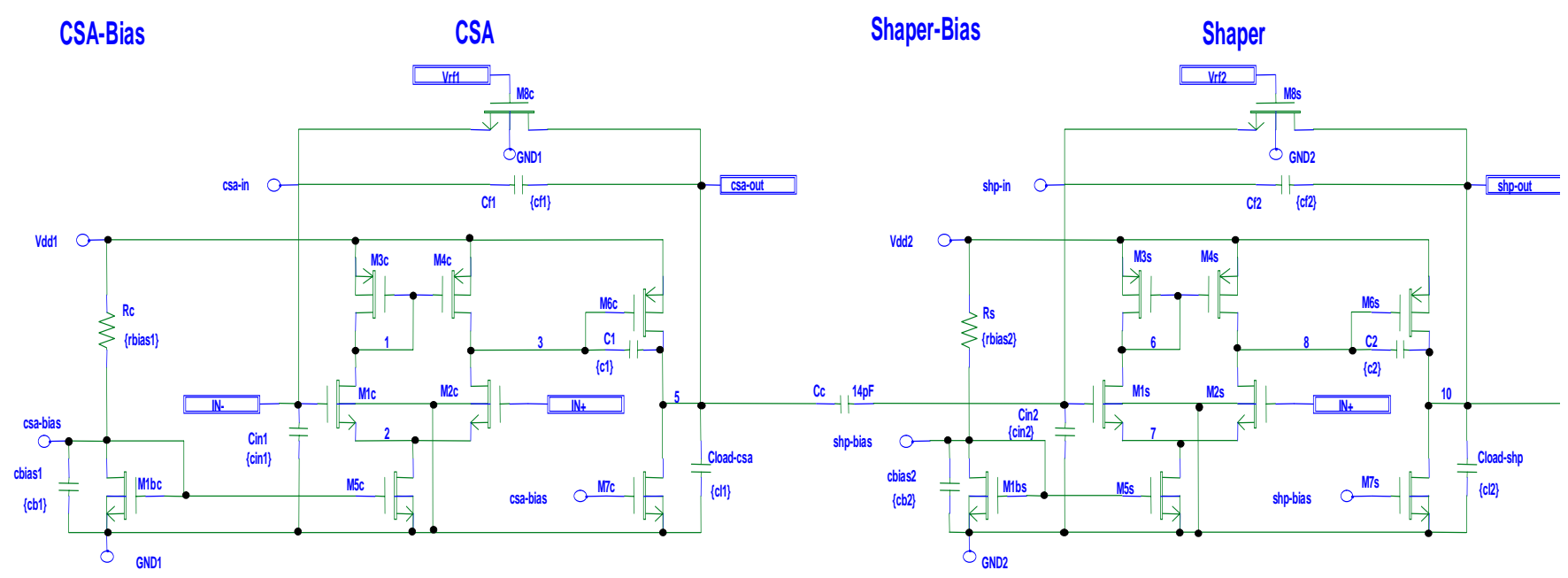

Figure 1. Electronic readout circuit of the channel including the integrator CSA and Shaper. 


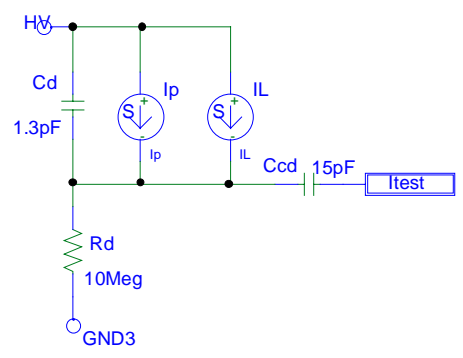

Figure 2. Model of a detector diode connected in AC.

Total noise is calculated by:

$$
E N C_{\text {tot }}^{2}=E N C_{t h}^{2}+E N C_{f}^{2}+E N C_{d}^{2}
$$

Optimized shaping time was calculated using the condition:

$$
\frac{d E N C_{t o t}}{d \tau}=0
$$

obtaining the value:

$$
\tau \cong C_{t} \sqrt{\frac{8 k T / 3 g_{m}}{2 q I_{L}}}
$$

The dependence of $E N C_{\text {tot }}$ on each of the parameters is calculated using a program written in "Mathematica" and is shown in Fig. 3. Table I indicates the optimized and used parameters in the circuit design and optimization.
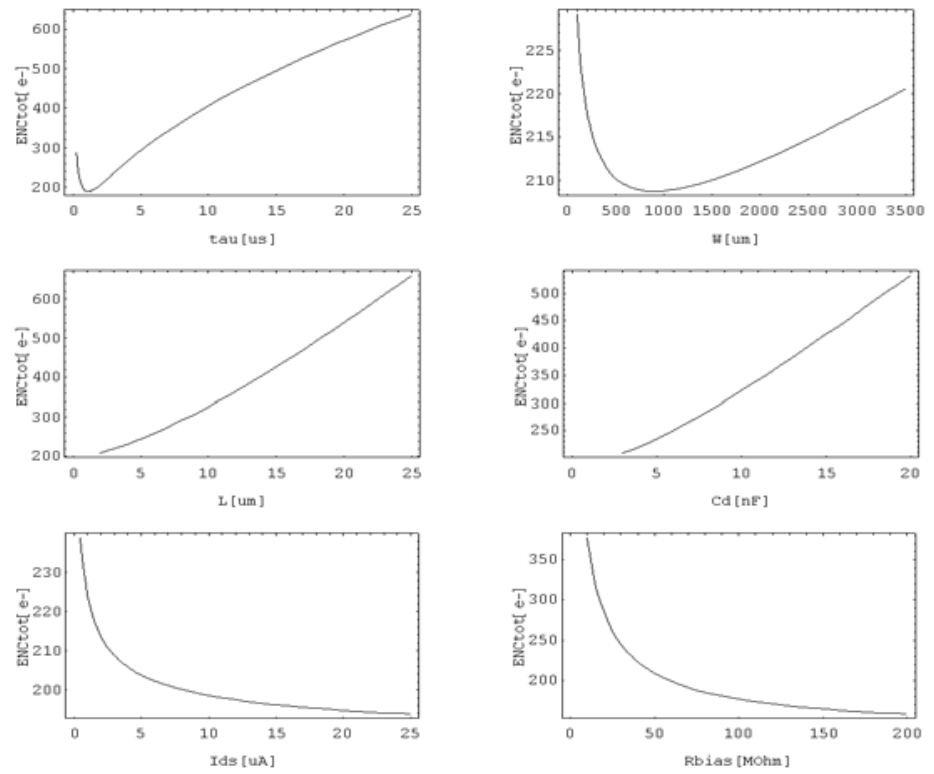

Figure 3. Dependence of ENC Cotal VS. all other parameters, using values reported in Table 1, for $T=300^{\circ} \mathrm{C}$. 
Table I. Optimized and other circuit parameters for ORBIT $2 \mu \mathrm{m}, \mathrm{N}$ well process.

\begin{tabular}{|l|l|l|l|}
\hline \multicolumn{1}{|c|}{ Parameter (description) } & Symbol & \multicolumn{1}{|c|}{ Value } & Unit \\
\hline Channel width of the input transistor of the CSA & $W$ & 788 & $\mu \mathrm{m}$ \\
Length width of the input transistor of the CSA & $L$ & 2 & $\mu \mathrm{m}$ \\
Lateral diffusion of the input transistor of the CSA & $L_{D}$ & 0.112 & $\mu \mathrm{m}$ \\
Current in the input transistor of the CSA & $I_{d s}$ & 3 & $\mu \mathrm{A}$ \\
Transconductance of the input transistor of the CSA & $g_{m}$ & 352.5 & $\mu \mathrm{A} / \mathrm{V}$ \\
Detector diode capacitance & $C_{d}$ & 1.3 & $\mathrm{pF}$ \\
Total capacitance at the CSA input & $C_{t}$ & 4.16 & $\mathrm{pF}$ \\
Leakage current in the detector diode & $I_{L}$ & 0.3864 & $\mathrm{pA}$ \\
Bias resistor for the AC connection in the detector diode & $R_{b i a s}$ & 50 & $\mathrm{M} \Omega$ \\
Feedback capacitance of the CSA & $C_{f}$ & 30 & $\mathrm{fF}$ \\
Feedback resistance of the CSA & $R_{f}$ & $>100$ & $\mathrm{M} \Omega$ \\
1/f technology process coefficient & $K_{f}$ & $1 \mathrm{x} 10^{-27}$ & $\mathrm{C} / \mathrm{m}^{2}$ \\
Time constant of the Shaper & $\tau$ & 2.1 & $\mu \mathrm{s}$ \\
Technology transconductance parameter & $K^{\prime}$ & 52.5 & $\mu \mathrm{A} / \mathrm{V}^{2}$ \\
Integrator order & $n$ & 1 & - \\
\hline
\end{tabular}

ENC rms noise for each of the contribution was:

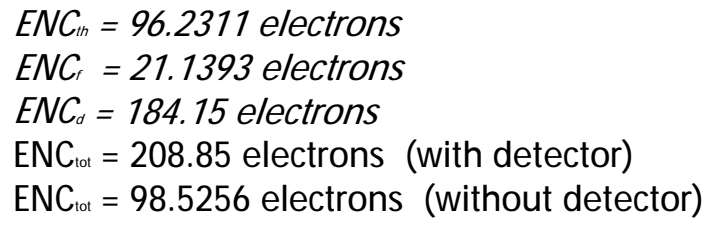

\section{NOISE MEASUREMENTS}

We used two different methods to measure the noise. In the first method the output waveform of the Shaper is stored and analyzed using a digital oscilloscope. Fig 4a shows the pulse characteristics, while Fig. $4 \mathrm{~b}$ shows noise at the output of the circuit without detector for an input capacitance of $C_{\text {in }}=3 p F$.
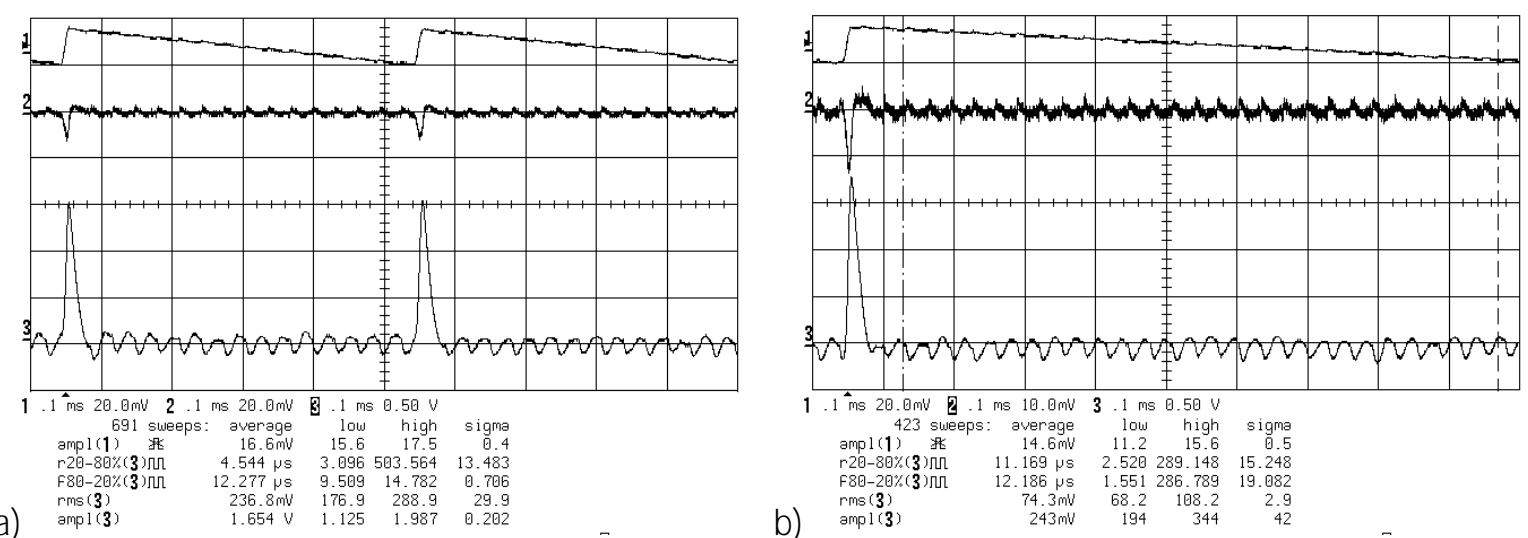

Figure 4. a) Curve (1)- CSA input signal equivalent to 3000 electrons; curve (2) - CSA output signal and curve (3)-

Shaper output signal. All points in the memory are included for mathematical processing. b) Curve (1) - CSA input signal equivalent to 3000 electrons; curve (2)- CSA output signal and curve (3)- Shaper output signal. Only the points inside the window were included for mathematical processing. 
ENC was calculated from amp/(3) in Fig.4a and $r m s(3)$ in Fig.4b using the following relations:

$$
E N C=\frac{(\text { ampl }(1)[\text { electrons }])(\mathrm{rms}(3))}{\operatorname{ampl}(3)}=\frac{3000 \text { electrons } \cdot 74.3 \mathrm{mV}}{1.654 \mathrm{~V}}=134 \text { electrons }
$$

Repeating the same for different values of input capacitance, points were traced and fitted to the equation:

$$
E_{\text {experim }}[\text { electrons }]=120+2.5 * C_{\text {in }}[\mathrm{pF}] \text {, }
$$

The second method used was to vary the amount of injected charge by changing the peak input voltage pulse applied through a capacitance equal to the CSA feedback capacitance. The output of the Shaper was connected to a counter with a fixed threshold voltage of $4 \mathrm{~V}$. The pulse generator was set in burst mode with 2000 pulses. The amount of pulses was counted for each voltage step and different input capacitance values indicated in Table 2.

The pulse amplitude vs Cin was plotted for each column in Table 2; differentiated and fitted to a gaussian function. The width of each adjusted function is a double sigma $(2 \mu)$ shown in Table 3 . The linear equation that better fits to all $\mu$ points can be expressed as:

$$
E N C_{\text {theor }}[\text { electrons }]=90+2.5 * C_{\text {in }}[\mathrm{pF}] \text {, }
$$

Table II. Input voltage pulses in [mV] vs. the amount of pulses counted, by the counter at the shaper output, for different input capacitance values in [pF].

\begin{tabular}{|c|c|c|c|c|c|c|c|c|c|}
\hline \multirow{2}{*}{$\begin{array}{c}\text { Pulse } \\
{[\mathrm{mV}]}\end{array}$} & \multicolumn{10}{|c|}{ Cin $[\mathrm{pF}]$} \\
\cline { 2 - 11 } & 0 & 3.4 & 6.02 & 9.86 & 17.3 & 26.7 & 39.6 & 47.4 & 55.6 \\
\hline 6 & 0 & 0 & 0 & 0 & 0 & 0 & 0 & 0 & 0 \\
\hline & 0 & 0 & 0 & 0 & 0 & 0 & 0 & 0 & 2 \\
\hline 8 & 0 & 0 & 0 & 0 & 1 & 1 & 4 & 7 & 33 \\
\hline 9 & 0 & 204 & & 69 & 157 & 186 & 171 & 157 & 276 \\
\hline & 1990 & 2000 & 1999 & 1986 & 1922 & 1661 & 1199 & 1043 & 1152 \\
\hline & 2000 & & 2 & 2000 & & 1995 & 1830 & 1774 & 1736 \\
\hline 12 & 2000 & 2000 & 2000 & 2000 & 2000 & 2000 & 1999 & 1992 & 1977 \\
\hline 13 & 2000 & 2000 & 2000 & 2000 & 2000 & 2000 & 2000 & 2000 & 2000 \\
\hline
\end{tabular}

Table III. Width of the gaussian curve (ENC rms) for each value of input capacitance [pF].

\begin{tabular}{|c|c|c|c|}
\hline Cin [pF] & $\begin{array}{c}\text { ENC }[\mathbf{m V}] \\
(\mathbf{2} \sigma)\end{array}$ & $\mathbf{( 2 \sigma )}$ & $(\sigma)$ \\
\hline 0 & 0.81776 & 163.552 & 81.776 \\
3.4 & 1.1946 & 238.92 & 119.46 \\
6.02 & 1.0514 & 210.28 & 105.14 \\
9.86 & 1.0222 & 204.44 & 102.22 \\
17.3 & 1.2045 & 240.9 & 120.45 \\
26.7 & 1.4591 & 291.82 & 145.91 \\
39.6 & 1.8779 & 375.58 & 187.79 \\
47.4 & 1.9731 & 394.62 & 197.31 \\
55.6 & 2.1545 & 430.9 & 215.45 \\
\hline
\end{tabular}


From both experimental methods used above to calculate the noise of the circuit we see that values are similar, that confirm the accuracy of measurements, and indicates correctness of theoretical optimizations, $E N C=98$ electrons against the experimental one $E N C=123$ electrons. The small difference can be attributed to circuit parasitic capacitances that change the filtering properties.

\section{CONCLUSIONS}

Theoretical calculations for noise optimization and experimental measurements of the noise in a low power, high output swing readout circuit for signal particle detection is presented, which ensure detection of as low as 400 electrons. Validity of the theoretical prediction is demonstrated using 2 methods to determine the experimental noise. The circuit presents noise as low as $E N C_{\text {tot }}=123$ electrons rms for $C_{d}=1.3 p F$ input capacitance while keeping a power consumption lower than $100 \mathrm{~W}$, and providing an output swing of $3 \mathrm{~V}$ which can be analyzed by laboratory equipment without other amplification stages.

\section{REFERENCES}

[1] Cerdeira A., Cuttin A., Fratnik F., Mutihac R. \& Colavita A., "Readout electronics in large detector matrix for soft X-ray in medical applications" Nuclear Instruments and Methods in Physics Research, Section A: Vol. 409 (1-3) 1998, pp. 497-500.

[2] Sansen W. \& Chang Z.Y., IEEE. Transactions on Circuits and System, Vol. 37 (11) 1990, pp. 1375.

[3] Chang Z.Y. \& Sansen W., Effect of $1 / f$ noise on resolution of CMOS analog readout system for microstrip and pixel detectors, Nuclear Instruments and Methods in Physics Research, Section A: Vol. 305 (8) 1991, pp. 553.

[4] Cicuttin A., Cerdeira A., Fratnik F., Colavita A. \& Vacchi A., , "Medusa-32: a low noise, low power silicon strip detector frontend electronics for space applications", Nucl. Instr. and Meth. in Phys. Res. A 390, 1997.

[5] Zhou C.Z. and Warburton K., "Noise Analysis of Low Noise, High Count Rate, PIN Diode X-Ray Detectors". IEEE Trans. On Nuclear Science. 43(3)1385, 1996.

[6] Arfelli F., Barbiellini G., Bonvicini V., Bravin A., Cantatore G., Castelli E., Cristaudo P., Di Michiel M., Longo R. \& Olivo A., "A digital readout system for the SYRMEP silicon strip detectors", INFN/TC-96/24, Nucl. Instr. and Meth. in Phys. Res. A 392, 1997.

Authors Byography

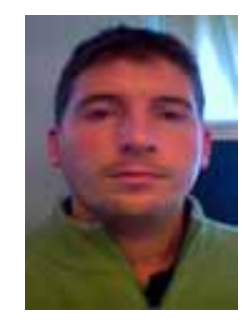

\section{Alberto Cerdeira Estrada}

Born in Habana, Cuba in the 1967, electro-physical engineer and master graduate from Moscow Energy Institute, Russia, in 1992. Doctorate in Sciences in the year 2000 in the IPN-CINVESTAV Mexico. He worked during 5 years in development of devices and integrated circuits for the nuclear physics, particularly the nuclear medicine and the astrophysics in the Habana Nuclear Center of Development, Cuba, and in the International Center of Physical Theoretician of Trieste, Italy. Among the works carried out there are a digital system of mammogram and a gamma rays telescope. Currently works for the DAI Telecom, Italy, in the design of new circuits integrated and teams for telecommunications, particularly, in cell phones GSM and UMTS. 


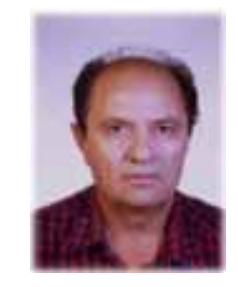

Adriano De Luca

Born in Foggia (Italy), graduate in Mathematics and Physics at Sto. Severo (Foggia). Doctorate in "Nucleonica and Automazione" at Milano (Italy). Member of the National System of Investigators since 1986. His was working during the first ten years in the ININ, Mexico, realizing various investigations and participating actively in the group that gained the National Prize of Applied Instrumentation in 1974. From 1982 to 1985 worked in the SGS Thompson in Milano (Italy). In 1991, he obtained USA Patent N. 07/483915 "Digital anode to determine the location of electrons in a given surface". In 1992, published the Text Book "Digital Systems" at Metropolitan Autonomous University, and is a writer of 87 articles.

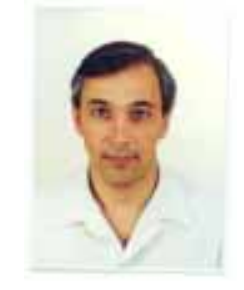

Andres Cuttin

Born in La Plata, Argentina December 2, 1961. He studied Physics at Exact Faculty Sciences of the University of La Plata where obtained the Bachelors degree in Physics in 1992. Since 1993 he works in the Microprocessor Laboratory which belongs to the International Center for Theoretical Physics, IAEA-UNESCO in Trieste, Italy. He collaborates regularly with the Italian National Institute of Nuclear Physics as associated investigator in the environment of the experimental physics of high energies. Currently works in the Experiment Compass of the CERN in the acquisition systems development and prosecution of data for gaseous detectors of elementary particles. Part of his activity is dedicated to physics and engineers training the third world in the field of the design of VLSI and logical devices programmable. He has published diverse scientific works related to the experimental physics and the instrumentation scientific development.

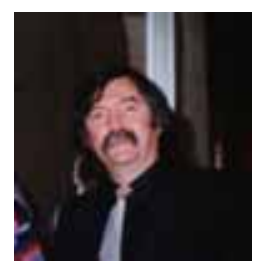

\section{R. Mutihac}

Born in Bucharest, Rumania, March 29 of 1952. He studied in the Physics Faculty at the Bucharest University, obtaining the Master title in Physics. In 1994 obtained, in the same University, the Ph.D in Biophysics. Currently works as Professor in the Department of Electricity and Biophysics, Faculty of Physics of the Bucharest University from the 2000. He published numerous scientific works in the fields of Neuronal Networks and Mathematical Modelling, Microelectronics, and Biophysics. His interest fields are: artificial intelligence, digital image processing and pattern recognition with Bayesian statistic. 